\title{
DIAGNÓSTICO SOBRE LAS ESTRATEGIAS PEDAGÓGICAS PROPUESTAS EN LOS CURSOS DE INVESTIGACIÓN DE LA UNIVERSIDAD NACIONAL ABIERTA Y A DISTANCIA - UNAD
}

\author{
Eliécer Pineda Ballesteros, Freddy Reynaldo Téllez Acuña \\ Fedra Lorena Ortiz Benavides, Dignora Inés Páez Giraldo y \\ Diana Patricia Landazábal Cuervo ${ }^{10}$
}

\begin{abstract}
RESUMEN
La realización de un diagnóstico sobre las estrategias pedagógicas propuestas en los cursos de investigación de la UNAD hace parte de un ejercicio que pretende diseñar una destreza de innovación tecno-pedagógica para la formación de la competencia investigativa en los cursos de esta área de investigación en dicha Universidad; para ello se eligió el curso "Trabajo de grado 201018" de la Escuela de Ciencias de la Educación y hemos diseñado y aplicado el formato de diagnóstico de cursos de investigación realizado por docentes auxiliares de la UNAD.
\end{abstract}

Al hacer la valoración del curso antes mencionado se encontraron más debilidades que fortalezas en el desarrollo de competencias investigativas y aquellas relacionadas con ámbito tecno pedagógico, en los siguientes aspectos a saber: variables humanas del tutor director, contenidos, actividades, intencionalidades formativas, coherencia institucional, evaluación, y como eje transversal, competencias en investigación.

A pesar de que los estudios profesionales y la experiencia investigativa del director de curso son sobresalientes, las intencionalidades formativas coherentes con el nivel de formación, alto grado de pertinencia con los contenidos del curso y contar con una especificación de actividades para el desarrollo de las competencias cognitivas relacionadas con la formación en investigación como observar, clasificar, distinguir, analizar, sintetizar, modelar, representar y comunicar, presenta debilidades marcadas en el aspecto tecno-pedagógico, inobservancia en las políticas de investigación derivadas del Sistema de Investigación de la Universidad Nacional Abierta y a Distancia- SIUNAD y los contenidos no tienen relación con la metacognición como elemento fundamental en el desarrollo de proyectos de investigación y especialmente en el proceso de formación en investigación.

Palabras clave: diagnóstico, estrategia, pedagogía, tecno pedagógico.

10 Grupos de investigación: GIUC: col0082304, Guane: col0026735, Gestión Vital: col0056646, Biotics: col0083633, Línea de investigación: Pedagogía, Didáctica y Currículo, Red de investigación: Visibilidad y gestión del conocimiento. E-mail: eliecer. pineda@unad.edu.co, freddy.tellez@unad.edu.co, fedra.ortiz@unad.edu.co, dignora.páez@unad.edu.co, diana.landazabal@ unad.edu.co 


\begin{abstract}
The accomplishment of a diagnosis of the pedagogic strategies proposed in the investigation courses of the UNAD, it does part of an exercise that tries to design a strategy of technopedagogic innovation for the formation of the research competence in the courses of this investigation area of UNAD; for this was chosen the course "201018 graduate work" at the School of Education Science and have designed and implemented the diagnostic format of the research course made by UNAD auxiliary teachers.

In making the above assessment of the course in the following aspects, director human Variables, content, activities, educational intentions, institutional coherence, evaluation, and as cross curricular: research competences, there were more weaknesses than strengths in the development research competences and those related to educational tech field.

Although professional studies and research experience of course leaders are outstanding, the educational intentions consistent with the level of education, high degree of relevance to the contents of the course and have a specification of activities to develop cognitive competences related to research training and as: observation, classifying, distinguish, analyzing, synthesize, modeling, representing and communicating, has marked weaknesses in the techno - pedagogical aspect, in research policies arising from the research system of the National Open University and Distance-Siunad and content that are unrelated to the cognition goal as a fundamental element in the development of research projects and especially in the process of research training.
\end{abstract}

Key words: diagnosis, strategy, education, techno - pedagogical

\title{
INTRODUCCIÓN
}

Aunque no existen estudios sobre la calidad de los procesos investigativos que desarrollan los estudiantes en la UNAD, en la comunidad académica se tiene la percepción de que los proyectos que ellos desarrollan tanto en pregrado como en posgrado tienen una baja calidad, con algunas excepciones que se destacan más por esfuerzos individuales, que como resultado del proceso educativo. Al parecer, y coincidiendo parcialmente con la opinión académica de la UNAD, el principal problema que contribuye para que se presente esta situación está asociado con la falta de competencias investigativas en los estudiantes que no escapan a los prejuicios del sistema educativo tradicional presencial.

En este ejercicio investigativo se ha tenido en cuenta entre otros autores a Machado et al., (2008) y Rodríguez et al., (2009) quienes exponen habilidades que son deseables en el proceso investigativo, a Barrera (2007), Fernández et al., ( 2001), Ried, Manjarrés (2007), Fonseca (2005), Cabrera (2006), quienes exponen propuestas alrededor de la formación de habilidades investigativas y a Fernández, et al., ( 2001), Balbis (s/f), Waite (2006), Gayol (2008) entre otros, los cuales plantean estrategias de formación en habilidades investigativas. 
El objetivo propuesto fue diseñar y aplicar un instrumento que permitiera evaluar un curso de investigación a la luz de los autores mencionados el cual posibilitó elaborar el diagnóstico sobre estrategias pedagógicas en los cursos de investigación, que intenta brindar un aporte a la calidad de estos cursos en la UNAD. Ante este panorama se espera, con esta ponencia, realizar un diagnóstico de las estrategias pedagógicas propuestas en los cursos de investigación de la UNAD.

\section{METODOLOGÍA}

La investigación es de tipo exploratoria. Se realizó un acercamiento al problema investigado, describiendo las tendencias conceptuales sobre el objeto de estudio y se analizó el conocimiento producido sobre competencias de investigación y estrategias para el desarrollo de las mismas construyendo un estado de arte. Luego, se elaboró un instrumento, se eligió un curso de investigación y se hizo la valoración de mismo a partir del instrumento.

\section{Elaboración del instrumento}

En la elaboración del instrumento se tuvieron presentes aspectos como: variables, dimensiones, indicadores, condiciones, porcentaje y el valor del indicador. Las variables tenidas en cuenta en la valoración fueron: variables humanas del tutor director, contenidos, actividades, intencionalidades formativas, coherencia institucional, evaluación y, como eje transversal, competencias en investigación.

- Variables humanas del tutor director, contó con los siguientes indicadores: Experiencia en investigación y perfil del tutor, y con las condiciones.

- Contenidos contó con los siguientes indicadores: organización temática, didáctica y tecno-pedagógica.

- Actividades contó con los siguientes indicadores: tipo de actividades y estrategias pedagógicas.

- Intencionalidades formativas contó con los siguientes indicadores: propósitos, objetivos, competencias y metas

- Coherencia institucional contó con los siguientes indicadores: identificación con la cultura Unadista de investigación, promoción de la participación en el SIUNAD.

- Coherencia en el eje de investigación contó con los siguientes indicadores: Diferenciación temática con otros cursos de investigación, diferenciación la competencias propuestas en los cursos de investigación.

- Evaluación contó con los siguientes indicadores: tipos de evaluación.

- Competencias en investigación, este se convirtió en un eje transversal, ya que se involucró con todas las dimensiones, contó con los siguientes indicadores:

- Competencias de investigación del tutor, contenidos para el desarrollo de competencias de investigación, formulación de competencias de investigación en las intencionalidades formativas, formulación de actividades para el desarrollo de competencias de investigación. 
Tabla 1. Valoración instrumento de evaluación

\begin{tabular}{|c|c|c|c|}
\hline \multirow{4}{*}{$\begin{array}{l}\text { Número de publicaciones } \\
\text { en revista indexada. }\end{array}$} & $\begin{array}{l}\text { El tutor/director ha publicado en } 3 \text { o más } \\
\text { revistas indexadas }\end{array}$ & 100 & \multirow{4}{*}{100} \\
\hline & $\begin{array}{l}\text { El tutor/director ha publicado en } 2 \\
\text { revistas indexadas }\end{array}$ & 66 & \\
\hline & $\begin{array}{l}\text { El tutor/director ha publicado en } 1 \\
\text { revista indexada }\end{array}$ & 33 & \\
\hline & $\begin{array}{l}\text { El tutor/director no ha publicado en } \\
\text { revistas indexadas }\end{array}$ & 0 & \\
\hline
\end{tabular}

Los indicadores a su vez contaron con unas condiciones que tenían un porcentaje con la siguiente escala $100,66,33$ y 0 . Cien (100) si cumplía con todo lo que estaba allí estipulado y cero (0) si no cumplía con nada de lo que estaba establecido, después de realizar la revisión se debía otorgar un valor al indicador.

Se eligió para la respectiva valoración por parte de los investigadores el curso Proyecto de grado 207018 de la ECE, seguidamente se realizó su aplicación.

Tabla 2. Fragmento del instrumento de evaluación

\begin{tabular}{|c|c|c|c|}
\hline Variables & Dimensiones & Indicadores & Valor \\
\hline \multirow{8}{*}{ Tutor } & \multirow{4}{*}{ Experiencia en Investigación } & $\begin{array}{l}\text { Número de grupos de investigación al } \\
\text { cual pertenece. }\end{array}$ & 100 \\
\hline & & Número de investigaciones realizadas. & 100 \\
\hline & & $\begin{array}{l}\text { Número de publicaciones en revista } \\
\text { indeexada. }\end{array}$ & 100 \\
\hline & & $\begin{array}{l}\text { Número de publicaciones en revista no } \\
\text { indexada. }\end{array}$ & 100 \\
\hline & \multirow{4}{*}{$\begin{array}{l}\text { Competencias de } \\
\text { investigación del tutor }\end{array}$} & $\begin{array}{l}\text { Domina conceptos de ciencia, tecnología } \\
\text { y técnicas }\end{array}$ & 100 \\
\hline & & $\begin{array}{l}\text { Reconoce el proceso que se debe realizar } \\
\text { para la elaboración de un proyecto de } \\
\text { investigación }\end{array}$ & 100 \\
\hline & & $\begin{array}{l}\text { Maneja tecnologías de información } \\
\text { y comunicación y software para el } \\
\text { desarrollo de la investigación }\end{array}$ & 66 \\
\hline & & $\begin{array}{l}\text { Muestra experiencia y uso de estrategias } \\
\text { pedagógicas para el acompañamiento } \\
\text { de los estudiantes en la formación de } \\
\text { investigación }\end{array}$ & 100 \\
\hline
\end{tabular}




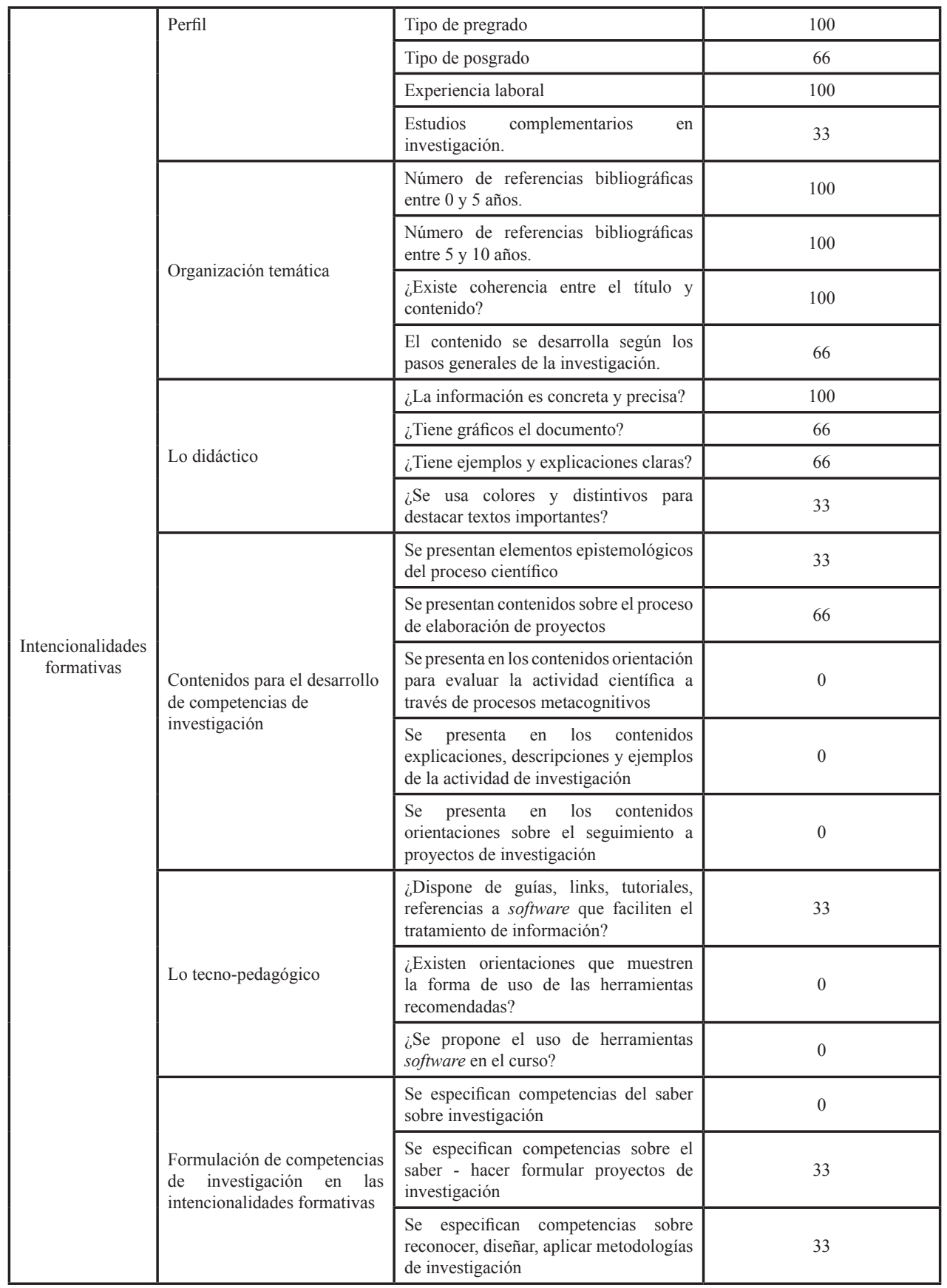




\begin{tabular}{|c|c|c|c|}
\hline & & $\begin{array}{l}\text { Se especifican las competencias } \\
\text { cognitivas relacionadas con la formación } \\
\text { en investigación como observar, } \\
\text { clasificar, distinguir, analizar, sintetizar, } \\
\text { modelar, representar, comunicar }\end{array}$ & 33 \\
\hline & & $\begin{array}{l}\text { Se especifican competencias del ser y } \\
\text { actitudinales en las intencionalidades } \\
\text { formativas }\end{array}$ & 100 \\
\hline \multirow{3}{*}{$\begin{array}{l}\text { Intencionalidades } \\
\text { formativas }\end{array}$} & \multirow{3}{*}{$\begin{array}{l}\text { Propósitos, Objetivos, } \\
\text { Competencias y Metas }\end{array}$} & ¿Están presentes? & 0 \\
\hline & & $\begin{array}{l}\text { ¿Son pertinentes de acuerdo con el } \\
\text { contenido? }\end{array}$ & 0 \\
\hline & & $\begin{array}{l}\text { ¿Existe coherencia con el nivel de } \\
\text { formación (pre o postgrado)? }\end{array}$ & 0 \\
\hline \multirow{14}{*}{ Actividades } & \multirow{5}{*}{ Tipos de actividades } & ¿Se propone revisión de artículos? & 0 \\
\hline & & ¿Existe estudio de caso? & 0 \\
\hline & & ¿Se proponen experimentos simulados? & 0 \\
\hline & & ¿Se debe realizar algún tipo de escrito? & 50 \\
\hline & & ¿Se diseñan fichas RAE? & 0 \\
\hline & \multirow{5}{*}{$\begin{array}{l}\text { Formulación de actividades } \\
\text { para el desarrollo de } \\
\text { competencias de investigación }\end{array}$} & $\begin{array}{l}\text { Se especifican actividades para el } \\
\text { desarrollo de competencias del saber } \\
\text { sobre investigación }\end{array}$ & 100 \\
\hline & & $\begin{array}{l}\text { Se especifican actividades para el } \\
\text { desarrollo de competencias sobre el } \\
\text { saber - hacer formular proyectos de } \\
\text { investigación }\end{array}$ & 66 \\
\hline & & $\begin{array}{l}\text { Se especifican actividades para el } \\
\text { desarrollo de competencias sobre } \\
\text { reconocer, diseñar, aplicar metodologías } \\
\text { de investigación }\end{array}$ & 66 \\
\hline & & $\begin{array}{l}\text { Se especifican actividades para el } \\
\text { desarrollode las competencias cognitivas } \\
\text { relacionadas con la formación en } \\
\text { investigación como observar, clasificar, } \\
\text { distinguir, analizar, sintetizar, modelar, } \\
\text { representar, comunicar }\end{array}$ & 100 \\
\hline & & $\begin{array}{l}\text { Se especifican actividades para el } \\
\text { desarrollo de competencias del ser y } \\
\text { actitudinales en las intencionalidades } \\
\text { formativas }\end{array}$ & 66 \\
\hline & \multirow{4}{*}{ Estrategias pedagógicas } & Actividades basadas en problemas & 0 \\
\hline & & Actividades basadas en proyectos & 100 \\
\hline & & Actividades de argumentación & 100 \\
\hline & & $\begin{array}{l}\text { ¿Son suficientes las actividades para } \\
\text { el desarrollo de las competencias } \\
\text { propuestas? }\end{array}$ & 50 \\
\hline
\end{tabular}




\begin{tabular}{|c|c|c|c|}
\hline \multirow{5}{*}{ Evaluación } & \multirow{5}{*}{ Tipos de evaluación } & ¿Hay co-evaluación? & 0 \\
\hline & & ¿Hay hetero-evaluación? & 66 \\
\hline & & ¿Hay auto-evaluación? & 0 \\
\hline & & ¿hay evaluación formativa? & 33 \\
\hline & & ¿Hay evaluación sumativa? & 100 \\
\hline \multirow{5}{*}{$\begin{array}{l}\text { Co h e r e n c i a } \\
\text { Institucional }\end{array}$} & $\begin{array}{l}\text { Identificación con la cultura } \\
\text { Unadista de investigación. }\end{array}$ & $\begin{array}{l}\text { ¿El curso es coherente con la misión } \\
\text { y visión de la UNAD en cuanto a } \\
\text { investigación? }\end{array}$ & 33 \\
\hline & \multirow{4}{*}{$\begin{array}{l}\text { Promoción de la participación } \\
\text { en el SIUNAD. }\end{array}$} & $\begin{array}{l}\text { ¿Los trabajos propuestos se inscriben } \\
\text { dentro de las temáticas de las redes de } \\
\text { investigación de la UNAD? }\end{array}$ & 0 \\
\hline & & $\begin{array}{l}\text { ¿Se promueve la participación en los } \\
\text { grupos y semilleros de la UNAD? }\end{array}$ & 0 \\
\hline & & $\begin{array}{l}\text { ¿Se promueve el uso de los formatos de } \\
\text { gestión de proyectos del SIUNAD? }\end{array}$ & 0 \\
\hline & & Valoración & $50 \%$ \\
\hline
\end{tabular}

\section{RESULTADOS}

A partir de la construcción del instrumento y su confrontación con el estado de arte se realizó la aplicación al curso de investigación Proyecto de grado 207018 de la Escuela de Ciencias de la Educación - ECE y se hizo la valoración del mismo, como se observa a continuación.

\section{Variables humanas del tutor/ director}

Se puede apreciar la necesidad de contar con la disponibilidad de la hoja de vida o del CVLAC del tutor, ya que la información suministrada en el perfil del curso es muy limitada.

Tanto los estudios profesionales como la experiencia en investigación son sobresalientes y, por lo tanto, adecuados para desempeñar funciones de director y tutor del curso, así como para participar dentro de redes académicas de investigación.

\section{Contenidos}

En este aspecto se tuvieron en cuanta para su valoración los siguientes ítems: organización temática, didáctica y tecno-pedagógica.

En relación con la organización temática, en el módulo se encontró que posee diversidad de fuentes bibliográficas; en el curso el director propone más de 10 referencias bibliográficas, que enriquecen el contenido del modulo. También se pudo verificar que hay coherencia entre el título y el contenido y el contenido se desarrolla consecuentemente con la mayoría de los lineamientos generales propuesto en la metodología de la investigación. De acuerdo con la revisión realizada se puede constatar que hay claridad en la organización temática y el desarrollo metodológico del curso. 
Respecto al tema que atañe a lo didáctico se pudo encontrar, en la revisión, que la información se presenta de forma concreta y precisa, con pertinencia para la elaboración de un trabajo de grado. A pesar de que posee algunos gráficos, es necesario implementar más el uso de estos en el curso, en algunos capítulos se registra que la información brindada es ilustrada con ejemplos y explicaciones claras. La información presenta escasez de colores y distintivos para destacar textos.

En el aspecto tecno-pedagógico nota claramente un gran vacío en el curso. La información presenta pocos elementos que facilitan el tratamiento de la información mediante enlaces, tutoriales, referencias a software. Se proponen herramientas como el c-map tools, pero no se da ninguna orientación de cómo utilizarlo.

\section{Las actividades}

En lo que respecta a las actividades se revisaron los siguientes ítems: tipos de actividades, formulación de actividades para el desarrollo de competencias de investigación y estrategias pedagógicas, y se encontró lo siguiente:

Dentro de las actividades verificadas se halló que el curso no ofrece posibilidad de desarrollo alrededor de la revisión de artículos científicos, el estudio de caso, los experimentos simulados (teniendo en cuenta que es un curso virtual) y el diseño de fichas RAE. Se evidencia, además, la construcción de un ensayo como punto de partida para el desarrollo de la propuesta de investigación.

En lo que respecta a la formulación de actividades para el desarrollo de competencias de investigación se encontró principalmente que en el curso se especifican algunas actividades que promueven el desarrollo de competencias del saber sobre investigación especialmente relacionadas con la lectura de textos y la recreación de los mismos mediante escritos elaborados por los estudiantes. Se evidenció una mediana participación en lo referente a la especificación de actividades para el desarrollo de competencias sobre el saber - hacer - formular proyectos de investigación. Si bien es cierto que los estudiantes trabajan la formulación de proyectos no es muy fuerte el trabajo encaminado a la formulación de problemas de investigación; de igual manera, es mediano en nivel de especificación de actividades que desarrollen competencias sobre reconocimiento, diseño y aplicación de metodologías de investigación. Al hacer el recorrido por el curso se puede verificar que hay una especificación de actividades para el desarrollo de las competencias cognitivas relacionadas con la formación en investigación como observar, clasificar, distinguir, analizar, sintetizar, modelar, representar y comunicar. Finalmente, hay que mencionar que si bien es cierto que se especifican algunas actividades para el desarrollo de competencias del ser y competencias actitudinales en las intencionalidades formativas, no es muy clara la forma como se pretenden llevar a cabo, pues los foros destinados para tal fin parecieran no cumplir con su propósito dada la notoria falta de participación en los mismos por parte de los estudiantes. 
En cuanto a las estrategias pedagógicas hay presentes en el curso algunas actividades basadas en proyectos y en actividades de argumentación, pero es latente la ausencia de actividades basadas en problemas y una mediana coherencia entre actividades y competencia.

\section{Intencionalidades formativas del curso}

El curso presenta dentro de su Protocolo Académico tres de las cuatro Intencionalidades Formativas solicitadas en los estándares de la Universidad. Falta la elaboración de las Metas propuestas dentro del proceso formativo. Dichas Intencionalidades Formativas presentan coherencia con el nivel de formación y un alto grado de pertinencia con los contenidos del curso.

Se recomienda, finalmente, hacer visible el link del Protocolo del Curso, ya que en el momento está oculto y, por lo tanto, no puede ser consultado por los estudiantes.

\section{Coherencia Institucional}

Este aspecto del instrumento busca identificar si se da una alta correlación entre las políticas de la UNAD en cuanto a la gestión de la investigación y el curso per se.

Se valoraron dos aspectos a saber: la identificación con la cultura Unadista de investigación y la promoción de la participación en el SIUNAD.

Con respecto al primer ítem se encontró que el curso escasamente promueve la educación para todos a través de la modalidad abierta y a distancia y la investigación en tanto es un curso en la modalidad de educación a distancia con propósitos de formar personas en la tarea de realizar su trabajo de grado.

Al revisar el segundo ítem no es posible identificar los trabajos que se proponen en el curso dentro de las temáticas generales de alguna red de investigación de la UNAD. Salvo por el elemento trasversal de la investigación, hay una completa inobservancia de las políticas de investigación que se derivan del SIUNAD; en este mismo sentido no hay un interés manifiesto en el curso por promover en los estudiantes la participación tanto en grupos como en semilleros de investigación.

\section{La evaluación}

Los tipos de evaluación que se propusieron fueron: autoevaluación, heteroevaluación y coevaluación, cómo también la evaluación sumativa y formativa.

De acuerdo con el formato de evaluación se reportan los siguientes resultados:

- Coevaluación: no se propicia el espacio para la co-evaluación entre el grupo de estudiantes

- Heteroevaluación: se presentan rúbricas de evaluación en las diferentes actividades, pero estas no presentan criterios claros en coherencia con las actividades propuestas 
- Autoevaluación: no se propicia el espacio para la auto evaluación en las actividades planteadas

- Evaluación formativa: la retroalimentación realizada por el tutor no es lo suficientemente fundamentada que le permita al estudiante continuar con el proceso de desarrollo cognitivo

- Evaluación sumativa: existe una escala de calificación y cada una de las actividades presentan un peso evaluativo claramente definido. La calificación máxima que se puede alcanzar en el curso es de 500 puntos.

- Evaluación por competencias: las actividades de evaluación no son coherentes con las competencias formuladas en las intencionalidades formativas del curso.

\section{Competencias de investigación}

Cuando se habla de competencias de investigación se pueden mencionar unas competencias básicas y unas avanzadas. Existen tres dimensiones en una competencia de investigación: la que se refiere al saber (epistemología de las ciencias), la del saber - hacer (destrezas y habilidades específicas para desarrollar un proceso investigativo y aquella del ser (actitudes y motivaciones) que están implicados en la tarea de investigación.

A continuación se presentará el resultado de la aplicación del instrumento a un curso de trabajo de grado, para observar lo referente a las competencias.

\section{Competencias del tutor}

Al realizar un análisis de las competencias del tutor se encontró experiencia en investigación, en la formulación, desarrollo de proyectos de investigación y divulgación de los resultados a través de la presentación de las ponencias y artículos. El resultado muestra la importancia de este aspecto en el acompañamiento a los estudiantes en la realización de su trabajo de grado y de la asignatura. Sin embargo, no es fácil evidenciar el perfil de los docentes a través de la plataforma, es necesario recurrir a la hoja de vida para ello y también es importante contrastar los resultados con otros cursos de investigación.

\section{Contenidos para el desarrollo de competencias de investigación}

Al observar el módulo del curso y medirlos con el instrumento diseñado se encontró que el curso no maneja elementos de epistemología es decir relacionados con el saber (conocimiento) sobre investigación, el concepto de ciencia o tecnología. Esta ausencia podría estar relacionada con la existencia de otros cursos que manejen esta temática epistemológica dentro de los programas, sin embargo, al observar el nuevo programa se detectó que sólo había dos cursos en el eje investigativo: el de Seminario de la Investigación y el de Trabajo de Grado, por lo cual se hace necesario introducir algunos elementos epistemológicos relacionados con el saber científico.

No se presentan contenidos que estén relacionados con la meta cognición como elemento fundamental en la elaboración y desarrollo de proyectos de investigación y especialmente 
en el proceso de formación de investigación. Algunos autores recomiendan que este proceso meta-cognitivo le permite al estudiante autorregular su propio aprendizaje y medir las metas que va alcanzando cuando está aprendiendo a escribir proyectos de investigación.

Frente al conocimiento que el estudiante requiere ir formando acerca de la formulación de proyectos de investigación, se encontró que se explica el proceso sin tener en cuenta el componente administrativo. Este componente referente a los recursos y la planeación del proyecto se constituye en un elemento importante del cual se carece en los contenidos del curso evaluado, salvo la presentación sobre lo que es un cronograma de trabajo.

En los contenidos no se presentan ejemplos sobre el diseño de un proyecto de investigación y su proceso, ni tampoco sobre la elaboración de un informe final, la divulgación de resultados y el seguimiento. Los ejemplos se constituyen en un elemento fundamental que permite al estudiante seguir algunos modelos en la elaboración de su propio trabajo. Los ejemplos y las gráficas son importantes para estimular otro tipo de inteligencia, que ayuda a afianzar el conocimiento y comparar con los propios productos de investigación.

Finalmente, no se encontraron explicaciones relacionadas con el seguimiento que se debe realizar a los proyectos, esto muestra que no se pretende desarrollar este tipo de competencias en estos cursos. Sin embargo, esta es una destreza muy importante en el momento de la ejecución del proyecto.

\section{Formulación de competencias de investigación en las intencionalidades formativas}

Al observar el protocolo del curso con relación a las competencias de investigación que se formulan, se ve que no existe claridad en la variedad de competencias que se deben desarrollar para poder alcanzar la realización de un trabajo de grado. Específicamente se encontró una o dos competencias relacionadas con el saber y con el saber hacer. También, de forma interesante, se encontró una gran concentración en las competencias de investigación relacionadas con el ser. Esto podría mostrar que existe una tendencia mayor en el curso a formar la motivación y el interés en la investigación que el desarrollar múltiples competencias relacionadas con el hacer o con el saber epistemológico.

Se considera que por ello es importante, al diseñar los cursos y tener en cuenta las intencionalidades formativas y los objetivos, tener claro un conjunto de competencias que se deben alcanzar en por lo menos tres dimensiones, de manera tal que contribuya con el diseño de las actividades y que esté muy relacionado con los contenidos del curso.

\section{Formulación de actividades para el desarrollo de las competencias de investigación}

Al revisar las actividades propuestas para el desarrollo de competencias, se encontró que las actividades están orientadas a generar saber específico sobre la ciencia, a desarrollar un proyecto de investigación desde la formulación del proyecto y lo metodológico y que, además, se cuenta con actividades que favorecen el desarrollo de competencias relacionadas con el ser. 
Sin embargo, el porcentaje de calificación estuvo principalmente en el 66\% lo cual indica que existe la necesidad de plantear otro tipo de actividades que permitan desarrollar más ampliamente las competencias en las tres dimensiones. Se observó sólo un 100\% en las actividades relacionadas con la actividad cognitiva como analizar, argumentar, distinguir, etc.

\section{Evaluación por competencias}

Se encontró que se evalúan algunas, no todas, de las competencias que se pretende formar a través del curso, no hay actividades para ello.

\section{CONCLUSIONES}

\section{Fortalezas}

Los estudios profesionales y la experiencia en investigación del director son sobresalientes, adecuados para desempeñar funciones de Director y Tutor del curso. De igual manera, son apropiados para participar dentro de redes académicas de investigación.

Con relación a los contenidos, existe coherencia y claridad entre el título, los contenidos y la metodología desarrollada en el curso. La información se presenta de forma concreta y precisa, con pertinencia para la elaboración de un trabajo de grado.

Se especifican algunas actividades que promueven el desarrollo de competencias del saber sobre investigación, especialmente relacionadas con la lectura de textos y la recreación de los mismos mediante escritos elaborados por los estudiantes.

Hay una especificación de actividades para el desarrollo de las competencias cognitivas relacionadas con la formación en investigación como observar, clasificar, distinguir, analizar, sintetizar, modelar, representar y comunicar.

Las intencionalidades formativas presentan coherencia con el nivel de formación y un alto grado de pertinencia con los contenidos del curso.

\section{Debilidades}

Con relación al ámbito tecno-pedagógico presentó pocos elementos que facilitan el tratamiento de la información mediante enlaces, tutoriales o referencias a software.

El curso no ofrece posibilidad de desarrollo alrededor de la revisión de artículos científicos, el estudio de caso, los experimentos simulados (teniendo en cuenta que es un curso virtual) y el diseño de fichas RAE.

No es muy fuerte el trabajo encaminado a la formulación de problemas de investigación. 
Aunque se especifican algunas actividades para el desarrollo de competencias del ser y competencias actitudinales en las intencionalidades formativas, no es muy clara la forma como se pretenden llevar a cabo éstas, pues los foros destinados para tal fin parecieran no cumplir con su propósito dada la notoria falta de participación en los mismos por parte de los estudiantes.

Respecto a las estrategias pedagógicas se puedo constatar que existen en el curso algunas actividades basadas en proyectos y en actividades de argumentación, pero es evidente la ausencia de actividades basadas en problemas y una mediana coherencia entre actividades y competencia.

No es posible identificar los trabajos que se proponen en el curso dentro de las temáticas generales de alguna red de investigación de la UNAD. Salvo por el elemento trasversal de la investigación, hay una completa inobservancia de las políticas de investigación que se derivan del SIUNAD; en este mismo sentido no hay un interés manifiesto en el curso por promover en los estudiantes la participación tanto en grupos como en semilleros de investigación.

Presenta muchas deficiencias en la variable Evaluación, por cuanto esta no se identifica como un proceso de motivación y orientación constante que les permita a los estudiantes alcanzar las intencionalidades formativas formuladas en el curso.

No maneja elementos de epistemología, es decir, relacionados con el saber (conocimiento) sobre investigación, el concepto de ciencia y tecnología.

No se presentan contenidos que estén relacionados con la meta cognición como elemento fundamental en la elaboración y desarrollo de proyectos de investigación y especialmente en el proceso de formación de investigación.

\section{PERSPECTIVAS DEL TRABAJO}

La labor pedagógico - investigativa es un acto que exige reflexiones y prácticas rigurosas sobre la función social y científica que desarrolla la Universidad, en ese sentido esta debe ofrecer los medios para ser posible la cristalización de experiencias investigativas en su comunidad académica que posibilite responder a un mundo globalizado en donde las tecnologías de la información y la comunicación TIC juegan un papel importante en los procesos de interacción social y aprendizaje.

Inquietos por la calidad investigativa al interior de la unad, un grupo de investigadores de la misma viene desarrollando un proyecto cuya intencionalidad es mejorar los procesos investigativos.

A través de esta ponencia se presenta un diagnóstico de las competencias investigativas a través de la valoración del curso Proyecto de grado, en el cual se pudieron constatar algunas fortalezas y debilidades frente a la generación de competencias investigativas y la orientación 
adecuada de la estrategias tecno-pedagógicas, es por ello que se hace pertinente generar una propuesta innovadora en la formación investigativa de los estudiantes, utilizando ambientes digitales, lo cual aportaría al desarrollo de la comunidad científica y académica en las universidades con modalidad de Educación a Distancia.

\section{REFERENCIAS BIBLIOGRÁFICAS}

ANDER-EGG. E. (1995), Técnicas de investigación social. Editorial Lumen, 24 a edición, Buenos Aires. págs. 50-52.

ARCHIBUGI, Daniele. (2004), Europa en la carrera de la innovación. Italia.

BALBIS Enrique. (s/f), La tarea investigativa. Disponible on line http://www.bibliociencias. cu/gsdl/collect/revistas/index/assoc/HASHed4f/56317009.dir/doc.pdf

BARRERA, Josefina. (2007), La enseñanza de la física a través de habilidades investigativas: una experiencia. Brasil.

BARRIGA, F. \& HERNÁNDEZ, G. (1999), Estrategias docentes para un aprendizaje significativo. Una interpretación constructivista. En: http://redescolar.ilce.edu.mx/ redescolar/biblioteca/articulos/pdf/estrate.pdf

BLAKE, Gary (1996). Quality in R \& S demands right writing. Research Technology Management: Mar/Apr. 39.2.

BARRETO ARGILAGOS, Gaspar; RUÍZ SOCARRÁS, José, y BLANCO SÁNCHEZ, Ramón. 'Necesidad y utilidad de la categoría 'competencia' en ciencias pedagógicas", en: Revista Iberoamericana de Educación, En: http://www.rieoei.org/deloslectores/2064Barreto.pdf. ISSN: 1681-5653

BARRERAJosefina.(2007),LaEnseñanza DeLaFísicaATravés DeHabilidades Investigativas: Una Experiencia, publicado en: https://www.researchgate.net/publication/26554669_La_ enseanza_de_la_Fsica_a_travs_de_habilidades_investigativas_una_experiencia 2007.

CADENAS, ROJAS, B., REYES, L, Competencias en el eje investigativo de la cohorte 2000-2004 del subprograma de maestría en la educación superior de la UPEL-IPB. En: http://www.inie.ucr.ac.cr/congreso/memoria/archivos/ponencias/yamelinecadenas.pdf.

CALDERÓN, A. y DE JORGE, M. Competencias en información y entorno tecnológico. En: http:// observatorio.cnice.mec.es/modules.php?op=modload\&name $=$ News\&file $=$ article $\&$ sid $=335$

CASTRO, Julia Adriana. (2006), La investigación en el pregrado o el metacurrículo. Colombia.

CASTELlANO, D. et al., (2002), Aprender y enseñar en la escuela. Una concepción desarrolladora. La Habana: Editorial Pueblo y Educación. 
CASTRO, J. (2006), La investigación en el pregrado o el metacurrículo. Universidad de Antioquia. En: http://viref.udea.edu.co/contenido/pdf/investigacion\%20metacurriculo.pdf

CASTELlANOS, D. et al. (2002), Aprender y enseñar en la escuela. Una concepción desarrolladora. La Habana: Editorial Pueblo y Educación.

CENDALES, Lola. (2003), Aprender a investigar, investigando. Venezuela.

FONSECA, G.; CHONA, G.; ARTETA, J.; IBÁÑEZ, X.; MARTÍNEZ, S. Y PEDRAZA, M. Estudio interpretativo sobre prácticas de enseñanza de profesores de ciencias experimentales, con relación al desarrollo de competencias científicas. (2005). Publicado en ENSEÑANZA DE LAS CIENCIAS, 2005. NÚMERO EXTRA. VII CONGRESO. Disponible en: http://www.google.com.co/url?sa=t\&source=web\&ct=res\&cd=1\&ved=0 CAYQFjAA\&url=http $\% 3 \mathrm{~A} \% 2 \mathrm{~F} \% 2 \mathrm{Fensciencias.uab.}$

DELORS, Jacques. (1996), La Educación Encierra un Tesoro. Santillana, Ediciones UNESCO.

DÍAZ BARRIGA, F. (1994), La formación en aspectos metacurriculares con alumnos de educación media superior, en: Perfiles Educativos, Vol. 65, Julio-Diciembre. P. 18

DUCOING, Patricia et al., (1988), La formación en investigación en el Colegio de Pedagogía. Un análisis metodológico en la perspectiva del plan de estudio", en Cuadernos del CESU, núm. 9, UNAM, México.

CABRERA Elier, (s/f,) La competencia investigativa del Profesor General Integral de Secundaria Básica en su formación inicial, disponible en http:/www.monografias.com/ trabajos38/competencia-investigativa/competencia-investigativa.shtml

GARCÍA, Carlos. (1998), Formación de habilidades investigativas en los profesionales de la actividad vial en Cuba. En http://www.monografias.com/trabajos42/formacion-cultural/ formacion-cultural2.shtml

GARCÍA, M. (1900), Aprendizaje por descubrimiento frente a aprendizaje por recepción. La teoría del aprendizaje verbal significativo, en: C; Col, J. Palacios y A marchesi (Eds), "Desarrollo psicológico y educación II, Madrid, Alianza.

GAYOL, María. (s/f), Competencias Investigativas. Su desarrollo en carreras del Área de la Salud. Argentina.

GAYOL María del Carmen, MONTENEGRO Silvana Marisa, TARRÉS María Cristina, D'OTTAVIO Alberto Enrique (2008), Competencias Investigativas. Su desarrollo en carreras del Área de la Salud. En: Revista Unipluriversidad / Facultad de Educación / Universidad de Antioquia. Disponible on line en: http://aprendeenlinea.udea.edu.co/ revistas/index.php/unip/article/view/950/823 
FONSECA, G.; CHONA, G.;ARTETA, J.; IBÁÑEZ, X.; MARTÍNEZ, S. y PEDRAZA, M. (s/f), Estudio interpretativo sobre prácticas de enseñanza de profesores de ciencias experimentales, con relación al desarrollo de competencias científicas. En: http:// ensciencias.uab.es/congres2005/material/comuni_orales/3_Relacion_invest/3_1/ Fonseca_320.pdf

GRAY, Crawford. (2007), Research Competencies Framework. Inglaterra

GONZALEZ, V. (2006), Educación y desarrollo de competencia profesionales en la Universidad. Su concepción e instrumentación desde una perspectiva socio-histórica del desarrollo humano. Trabajo presentado en Universidad en soporte digital.

HERNÁNDEZ, Lázaro, DÍAZ, C., BORROTO, M. (s/f), La formación de habilidades investigativas en los futuros ingenieros agrónomos. Un reto de la universidad Cubana. Cuba. En: www.contua.org/noticias/Prog_univ2010.pdf

HODSON, D., 1992b. Assessment of practica1 work: some considerations in Philosophy of Science, Science \& Education, 1(2), pp. 115-144.

HUERTA J., PÉREZ I. S., CASTELlANOS A. R. (2003), Desarrollo curricular por competencias profesionales integrales. En: http://educacion.jalisco.gob.mx/consulta/ educar /13/ 13Huerta.html

IRIGOIN M, VARGAS F. (2002), La formación basada en competencias. El diseño de la formación basada en competencias En: Competencia laboral: manual de conceptos, métodos y aplicaciones en el sector salud. (Módulo 2, unidad 5). Montevideo: CINTERFOR-OPS; 2002: 252.

IAFRANCESCO, G. (2003), La investigación en educación y Pedagogía. Bogotá: Ed. Magisterio.

LERMA, HD (2004), Metodología de la investigación: Propuesta, Anteproyecto y Proyecto, 3. a ed., Ecoe Ediciones, Bogotá.

LUCIO, Ricardo (1995), El constructivismo y la práctica pedagógica, en: Educación y Cultura. No. 36-37. Memorias Segundo Congreso Pedagógico Nacional, FECODE, Págs. 169-170

LUNDVALL, B. A. y BORRÁS, S., (1998): The Globalising Learning Economy: Implications for Innovation. 177 EKAINA•JUNIO 2009 ZERBITZUAN 45 Policy, European Commission, D.G. XII, Bruselas.

MACHADO, Evelio, MONTES, Nancy, MENA, Alodio, El desarrollo de habilidades investigativas como objetivo educativo en las condiciones de la universalización de 
la educación superior, publicado en: Revista Pedagogía Universitaria Vol. XIII No. 1 2008, disponible en: http://revistas.mes.edu.cu/Pedagogia-Universitaria/articulos/2008/ numero/189408108.pdf/view

MALDONADO, L et al., (2007), Gestión del conocimiento: Visibilidad del Desarrollo Científico. Colombia, Editorial: UNAD.

MALDONADO, Luis et al., (2007), Visibilidad y formación en investigación. Estrategias para el desarrollo de competencias investigativas. Colombia. Revista Studiositas. Bogotá (Colombia. 2(2): 43- 56 Abril-Junio de 2007. ISSN 19090366

MILLER GE (1990), The assessment of clinical skills/competences/performance. Acad. Med.Supplement 65: 565-567.

MORENO, María. (2005), Potencias la investigación. Un Currículum transversal de educación para la investigación. México.

NÚÑEZ ROJAS N. (2007), Revista Iberoamericana de Educación N. 44. Volumen 6. Desarrollo de habilidades para la Investigación Universidad Católica Santo Toribio de Mogrovejo, Perú.

PADILLA María Antonia, SURO Ana Lucía, Seguimiento de la adquisición de las competencias Científicas de investigadores en formación: datos Preliminares, publicado en: 2005 - Avances en la Investigación Científica en el CUCBA - Centro de Estudios e Investigaciones en Comportamiento Universidad de Guadalajara, Centro de Estudios e Investigaciones en Comportamiento - Universidad de Guadalajara, disponible en:http://www.cucba.udg.mx/new/publicaciones/avances/avances_2005/Biologia/ PadillaVargasMariaAntonia/PadillaVargasMariaAntonia(4).pdf, 2005

PÉREZ, Coralia y otros. Las habilidades e invariantes investigativas en la formación del profesorado. Una propuesta metodológica para su estudio. Perú.

PICÓN G. (1986), Un Perfil Deseable para el Egresado de Posgrado en la Venezuela de Hoy. Investigación y Postgrado Volumen 1 Nro. 4.

RESTREPO, Guillermo. (s/f), Una universidad hacia la sociedad del conocimiento.

RIZO, Martha. (s/f). Enseñar a investigar investigando. Experiencias de investigación en comunicación con estudiantes de la Licenciatura en Comunicación y Cultura. En: http:// historiageografiasecundaria.blogspot.com/ 